\title{
LV-OP-3-7
}

\section{Risk factors and impact of unplanned conversion during laparoscopic hepatectomy on oncologic outcomes in patients with hepatocellular carcinoma}

\author{
Hyojin SHIN, Jai Young CHO*
}

Department of Surgery, Seoul National University Bundang Hospital, Seongnam, Korea

Introduction: This study aimed (1) to compare the perioperative and oncologic outcomes of laparoscopic (Lap), unplanned open conversion (Conversion) and planned open (Open) liver resection for hepatocellular carcinoma (HCC) located in the anterolateral segments of the liver and (2) to identify the risk factors for unplanned open conversion.

Methods: We retrospectively reviewed the data of 374 patients who underwent liver resection for HCC located in anterolateral segments between 2004 and 2018. All patients were divided into three groups for analysis: Lap $(\mathrm{N}=299)$, Open $(\mathrm{N}=62)$ or Conversion $(\mathrm{N}=13)$.

Results: Compared to Lap group, Conversion group showed longer operation time (300.00 \pm 142.08 vs $182.03 \pm 105.65 \mathrm{~min}, p=0.000)$, greater blood loss $(1,596.15 \pm 1,604.87$ vs. $411.35 \pm 614.33 \mathrm{~mL}, p=0.021)$ with higher transfusion rate $(23.1 \%$ vs. $5.4 \%, p=0.009)$, higher postoperative complication rate $(38.5 \%$ vs. $12.4 \%, p=0.008)$, longer hospital stay (10.38 \pm 5.69 vs. $6.48 \pm 7.71$ days, $p=0.040)$. Moreover, Conversion group showed lower R0 resection rate $(84.6 \%$ vs. $96 \%, p=0.000)$ and higher early tumor recurrence rate $(61.5 \%$ vs. $23.7 \%, p=0.008$ ). Even in comparison with Open group, Conversion group showed longer operation time (300.00 $\pm 142.08 \mathrm{vs} 222.42 \pm$ $88.10 \mathrm{~min}, p=0.012)$ and greater blood loss (1,596.15 $\pm 1,604.87$ vs. $812.50 \pm 977.66 \mathrm{~mL}, p=0.024)$. Risk factors for unplanned conversion to open were analyzed as bigger tumor size (OR 1.35, 95\% CI 1.05-1.74, $p=0.020$ ), multiple tumor (OR 5.95, 95\% CI 1.45-24.39, $p=0.013$ ), and other organ invasion (OR 15.32, 95\% CI 1.80-130.59, $p=0.013$ ).

Conclusions: Unplanned open conversion showed poor perioperative and oncologic outcomes compared to laparoscopy and planned open hepatectomy. Therefore, planned open liver resection should be recommended for HCCs located in anterolateral liver segments. 